\title{
Acyl-Coenzyme A:Cholesterol Acyltransferase 2 (ACAT2) Is Induced in Monocyte-Derived Macrophages: In Vivo and In Vitro Studies
}

\author{
Naomi Sakashita, Akira Miyazaki, Catherine C. Y. Chang, Ta-Yuan Chang, \\ Emi Kiyota, Maki Satoh, Yoshihiro Komohara, Peter M. Morganelli, Seikoh Horiuchi, \\ and Motohiro Takeya
}

Second Department of Pathology (NS, EK, YK, MT), Department of Biochemistry (MS, SH), Kumamoto University School of Medicine, Kumamoto, and Department of Biochemistry (AM), Showa University School of Medicine, Tokyo, Japan; Department of Biochemistry (NS, CCYC, T-YC), Dartmouth Medical School, Hanover, New Hampshire; and Department of Microbiology (PMM), Dartmouth Medical School and Veteran's Administration Hospital, White River Junction, Vermont

\begin{abstract}
SUMMARY: To test the possibility that acyl-coenzyme A:cholesterol acyltransferase 2 (ACAT2) may be expressed in human macrophages under pathologic conditions, we employed specific anti-ACAT2 antibodies and found clear ACAT2 signals in lipid-laden as well as lipid-free macrophages under various disease conditions, including atherosclerosis. However, no ACAT2 signal was detectable in macrophages under normal physiologic conditions. Using cultured human macrophages derived from blood-borne monocytes, immunoblot and RT-PCR analyses demonstrated that immature macrophages expressed only ACAT1, but the fully differentiated macrophages expressed both ACAT1 and ACAT2. Furthermore, RT-PCR clearly revealed the presence of both ACAT1 and ACAT2 mRNAs in human atherosclerotic aorta. Double immunohistochemical staining indicated that in human atherosclerotic aorta, all macrophages expressed ACAT1, while approximately $70 \%$ to $80 \%$ of macrophages also expressed ACAT2. In congenital hyperlipidemic mice, immunohistochemistry and RT-PCR demonstrated that ACAT2 was also present in lipid-laden cells of the atheromatous plaques. Our results suggest that in atherosclerotic plaque, the ability of macrophage foam cell transformation may be augmented by the dual expressions of ACAT1 and ACAT2. Additional immunoblot and RT-PCR experiments showed that the ACAT2 signal was clearly detectable in thioglycollate-elicited exudate mouse macrophages but not in peritoneal resident macrophages. We conclude that under various pathologic conditions, fully differentiated macrophages express ACAT2 in addition to ACAT1. (Lab Invest 2003, 83:1569-1581).
\end{abstract}

$B$ iosynthesis of cholesteryl ester from cholesterol and long chain fatty acyl-coenzyme $A$ is one of the essential functions for mammalian cells to maintain their proper plasma membrane cholesterol content. This process is carried out by the enzyme acylcoenzyme A:cholesterol acyltransferase (ACAT) (Chang et al, 1997). ACAT activity is ubiquitously present in various mammalian cells and tissues. In addition to its role in intracellular cholesterol homeostasis, its major physiologic functions include lipoprotein assembly in the liver, dietary cholesterol absorption in the small intestine, and steroid hormone synthesis in the steroidogenic organs such as adrenal

\section{DOI: 10.1097/01.LAB.0000095687.17383.39}

Received July 11, 2003.

This work was supported by a Grant-in-aid from Japan Society for the Promotion of Science No. 12770637 (to NS) and by NIH research grant No. HL36709 (to T-YC).

Address reprint requests to: Dr. Naomi Sakashita, Department of Biochemistry, Dartmouth Medical School, 7200 Vail Building, Room 304, Hanover, New Hampshire 03755. E-mail: Naomi.Sakashita@ Dartmouth.edu and gonads (Chang et al, 1997). In addition, under hyperlipidemic conditions, the accumulation of cytoplasmic cholesteryl esters produced from ACAT in macrophages constitute the foam cell formation, which is a hallmark of early stage atherosclerosis (Tabas, 1995). For these reasons, pharmacologic inhibition of ACAT activity has been considered as an attractive drug therapy for lipid-lowering and for antiatherosclerosis (Matsuda, 1994). Currently, two ACAT isozymes, ACAT1 and ACAT2, have been identified in humans and in animals (Anderson et al, 1998; Cases et al, 1998; Chang et al, 1993; Oelkers et al, 1998). Previous reports indicate that ACAT1 is widely expressed in various types of cells and tissues (Pape et al, 1995). In particular, ample expression of ACAT1 protein in human atherosclerotic lesions and in cultured human monocyte-macrophages have been demonstrated (Miyazaki et al, 1998), supporting the idea that ACAT1 plays an important role in differentiating monocytes and in forming the macrophage foam cells. In contrast, the tissue distribution of ACAT2 is very restricted: ACAT2 $\mathrm{mRNA}$ and protein signals have only been demonstrated in enterocytes (Buhman 
et al, 2000; Chang et al, 2000; Lee et al, 2000; Oelkers et al, 1998) and to a certain extent in hepatocytes (Anderson et al, 1998; Cases et al, 1998; Chang et al, 2000, 2001; Joyce et al, 1999; Oelkers et al, 1998). In hepatocytes, among the three species examined thus far (human, monkey, and mouse), the relative distribution of ACAT1 and ACAT2 enzyme activities differ: while ACAT2 is the major isoenzyme in monkey and mouse hepatocytes, ACAT1 is the major isoform in human hepatocytes (Anderson et al, 1998; Cases et al, 1998; Chang et al, 2000; Lee et al, 1998; Oelkers et al, 1998). Thus, although there is strong evidence implicating the role of ACAT1 in macrophage foam cell formation and ACAT2 in intestinal cholesterol absorption, the relative functional roles of ACAT1 and ACAT2 in the hepatic lipoprotein assembly process remain to be clarified.

Until now the possible presence of ACAT2 in other tissues and cells has not been reported. Recently the ACAT1 gene knockout (ACAT1-/-) mice have become available (Accad et al, 2000; Yagyu et al, 2000). In ACAT1-/-:apolipoprotein E knockout (Apo E-/-) or ACAT1-/-:low density lipoprotein receptor knockout (LDLR-/-) mice, Yagyu et al (2000) documented that there were decreased but clearly detectable amounts of cholesteryl esters present within the atherosclerotic lesions (Yagyu et al, 2000). The origin of cholesteryl ester present in atheromatous plaques under an ACAT1 gene knockout condition remained unexplained. To address this issue, we tested the possibility that both ACAT1 and ACAT2 are expressed in macrophages under certain conditions. In this report, we demonstrated the in vivo presence of ACAT2 in both human and mouse macrophages under various pathologic situations, especially in atherosclerosis. We also used cell culture systems to demonstrate the presence of ACAT2 in macrophages under certain in vitro conditions.

\section{Results}

\section{ACAT2 Expression in Human Tissues under Various Pathologic Conditions}

We have previously shown that the rabbit polyclonal anti-ACAT2 antibody, DM54, specifically recognized the ACAT2 protein; on immunoblots, it gave a single 46-kd band when extracts prepared from various ACAT2-expressing human cells were employed (Chang et al, 2000). In this report we used DM54 in a highly sensitive immunostaining method (Envision Plus) to obtain a specific immunohistochemical reaction to the ACAT2 protein in human tissues. The results presented in Figure 1 clearly demonstrate that ACAT2 is localized in the apical portion of the intestinal villi (Fig. 1A, with lower magnification) and is concentrated at the brush border region of the enterocytes (Fig. 1B, with higher magnification). These results are consistent with the ACAT2 localization data previously demonstrated in nonhuman primate intestines (Lee et al, 2000). To serve as a negative control, nonimmunized rabbit Ig did not give any positive reaction (Fig. 1C). Histologic analysis on pathologic tissue samples revealed that positive ACAT2 signals were detected in macrophages during cholesterolosis in the gall bladder (Fig. 1D). Clearly positive signals were also observed in macrophages present during enteral xanthoma (Fig. 1E), granulomatous lymphadenitis (Fig. 1F), foreign-body granuloma arising from cutaneous epidermoid cyst (Fig. 1G), as well as during eyelid xanthoma, proliferating synovitis, tuberculosis of the skin, and during pulmonary sarcoidosis (data not shown). All of these ACAT2-expressing cells were co-stained by the macrophage-specific antibody antiCD68 (KP1) (Fig. 1I). Double immunohistochemical stainings using DM54 and KP1 clearly demonstrated that the CD68-positive macrophages express ACAT2 in serial sections from pathologic human tissues (Fig. $1, \mathrm{H}$ to J). Interestingly, although there were many CD68- and ACAT1-positive tissue resident macrophages (Kupffer cells) in the normal human liver (Chang et al, 2000; Sakashita et al, 2000), no ACAT2 signal was detected in the liver (data not shown). Similarly, macrophages under physiologic conditions such as sinus macrophages in the spleen and lymph nodes did not react to DM54 at all (data not shown). These results imply that ACAT2 expression in macrophages is detectable but only under various pathologic conditions.

\section{ACAT2 Expression in Cultured Human Monocytes and Macrophages}

To explore the nature of ACAT2-positive macrophages, we employed an in vitro culture system to monitor the expressions of both ACAT1 and ACAT2, using the blood-borne monocytes-macrophage lineage (Johnson et al, 1977; Zuckerman et al, 1979). We had previously used the same cell culture system to monitor ACAT1 expression (Miyazaki et al, 1998). In immunoblot, the ACAT1-specific antibodies recognize a single 50-kd band, while the ACAT2-specific polyclonal antibodies recognize a single 46-kd band (Fig. 2A). The ACAT1 expression was significantly upregulated during the early phase of differentiation in human monocytes and macrophages, confirming our previous work (Miyazaki et al, 1998), and identical $50-k d$ signals were seen in positive control cell lines, hACAT1, stable clone of $\mathrm{CHO}$ cells that expressed human ACAT1 (Chang et al, 1998, 2000), and Caco2 cells (Chang et al, 1995, 1998). In contrast to ACAT1, ACAT2 expression was significantly up-regulated during the late stage of cell differentiation. A 46-kd signal corresponding to those from ACAT2 in differentiated human macrophages was shown in $\mathrm{Caco} 2$ cells, whereas major $\sim 50-k d$ histidine-tagged human ACAT2 signal and minor $\sim 46-\mathrm{kd}$ human ACAT2 signal were detected in HisACAT2 cells (stable clone of $\mathrm{CHO}$ cells that expressed histidine-tagged human ACAT2). Importantly, there was no ACAT2 signal in hACAT1 cells in immunoblot, indicating no immunologic crossreaction between DM54 and human ACAT1 protein. In nine separate experiments, we have consistently 

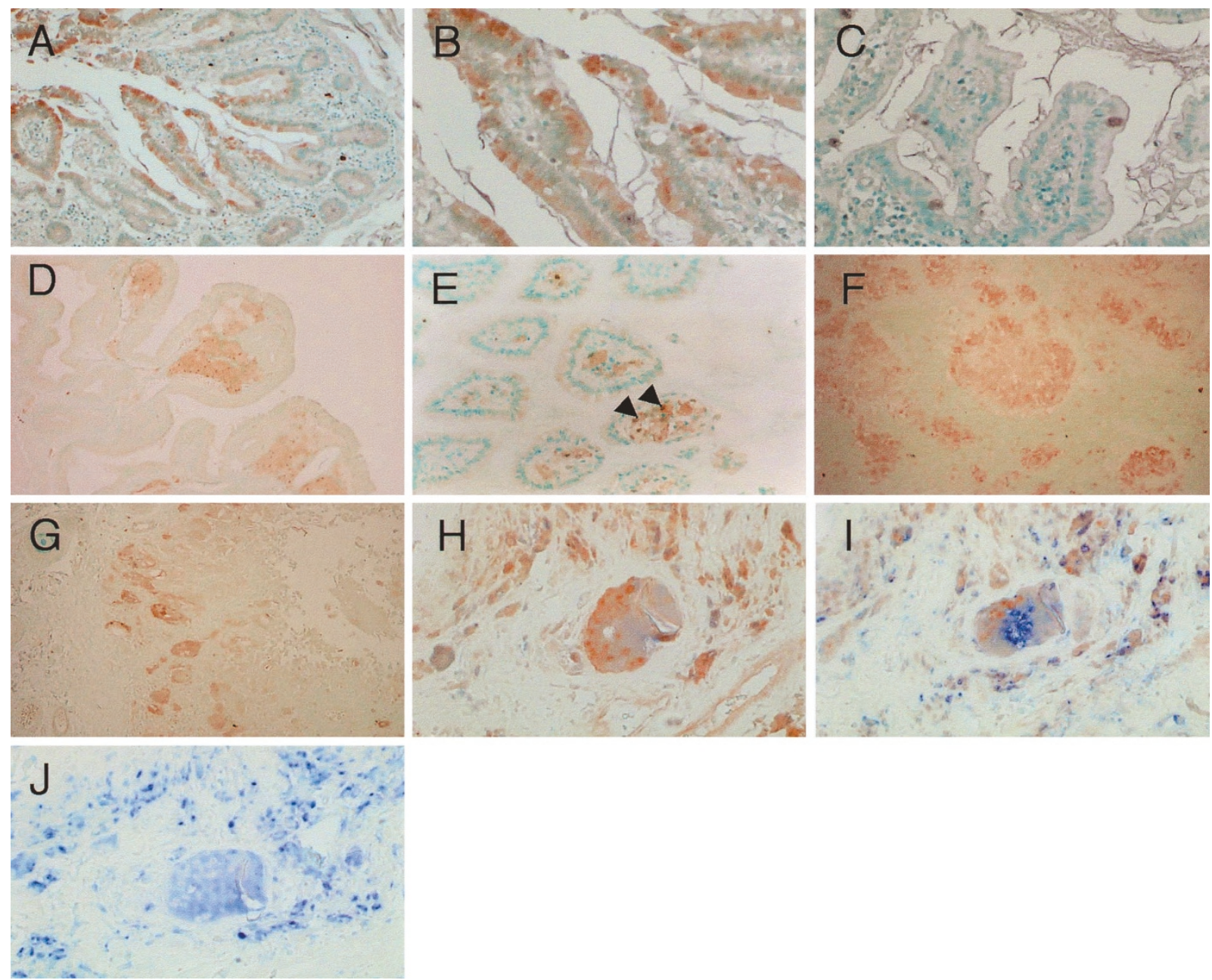

Figure 1.

Immunohistochemical demonstration of acyl-coenzyme A:cholesterol acyltransferase 2 (ACAT2) (brown color) in various human tissues. Immunohistochemistry using anti-ACAT2 antibody DM54 revealed that ACAT2 was detected in the apical region of the enterocytes in normal human small intestine (A); the same data viewed under higher magnification demonstrated that ACAT2 signals were localized in the brush border region (B). Negative control on serial sections of $A$ and $B$ using nonimmunized rabbit Ig gave no signal $(C)$, indicating the specificity of the immunoreaction in $A$ and $B$. ACAT2 signals were detected in foamy macrophages during cholesterolosis in gall bladder (D) and in enteral xanthoma (E, arrowheads). ACAT2 signals were also detected in macrophages accumulated in epithelioid granulomas, granulomatous lymphadenitis (F), and foreign-body granuloma of ruptured epidermoid cyst of the skin (G). In serial sections of foreign body granuloma from postoperative granulation tissue ( $\mathrm{H}$ to J), many CD68-positive macrophages as well as CD68-positive multinucleated giant cell (blue color, I and J) expressed ACAT2 (brown color, $\mathrm{H}$ and I). Objective lenses used were $\times 20$ in $\mathrm{C}, \times 10$ in $A$ and $D$ to $F, \times 40$ in $B$ and $G$ to I. For samples used in $D$, F, and $G$ to J, tissue sections were obtained from paraffin-embedded tissue samples derived from surgical specimens.

found that levels of ACAT1 protein reached maximal levels on or before Day 7, but those of ACAT2 reached plateau on Day 10 or later. Expressions of both ACAT1 and ACAT2 in differentiated macrophages were confirmed by immunocytochemical staining (Fig. 2C). In addition, by RT-PCR analysis, the mRNA of ACAT1 was detected in every human monocyte-derived macrophage examined as well as positive control cell lines, whereas ACAT2 mRNA was demonstrated on Day 7 or later differentiated macrophages and positive controls (Fig. 2B). Interestingly, by nested RT-PCR experiments, we observed ACAT2 signals in human macrophages isolate at Day 1 from three out of eight individuals (data not shown), suggesting that in some individuals very low ACAT2 mRNA expression may already be present in the early stage of differentiation of the macrophages.

\section{Detection of ACAT2-Positive Cells in Human Atherosclerotic Aorta}

We had previously demonstrated ample presence of ACAT1 in macrophages present in human atherosclerotic lesions (Miyazaki et al, 1998). To examine the possible presence of ACAT2 in the lesions, we used specific antibodies against ACAT1 or ACAT2, respectively, to perform immunostainings. The results revealed that there were numerous ACAT1- and ACAT2positive cells in the aorta with atherosclerosis (Fig. 3); the numbers of ACAT1- and ACAT2-positive cells increased significantly with the progression of atherosclerosis. We examined 15 different specimens and found that in every specimen examined, the number of ACAT2-positive cells was invariably fewer than that of ACAT1-positive cells. Most of the ACAT1- or ACAT2- 


\section{A immunoblot}

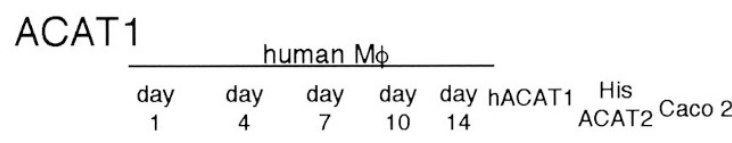

148 kDa

$98 \mathrm{kDa}$

64 kDa

50 kDa

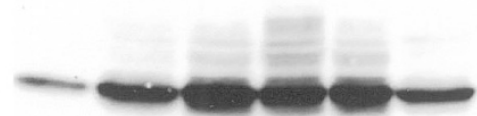

$36 \mathrm{kDa}$

22 kDa

\section{B RT-PCR}

ACAT1

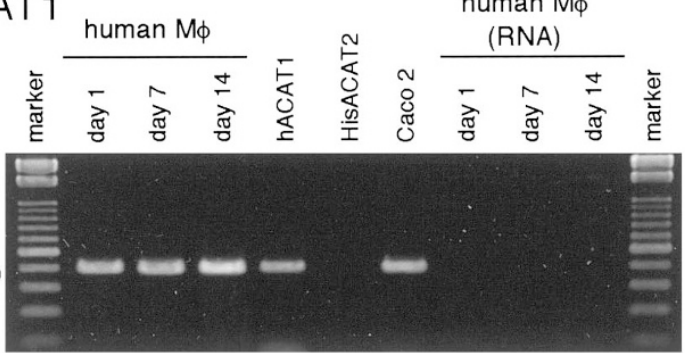

ACAT2

\begin{tabular}{ccccc}
\multicolumn{6}{c}{ human $\mathrm{M} \phi$} \\
\hline day & day & day & day & day hACAT1 His \\
1 & 4 & 7 & 10 & 14
\end{tabular} ACAT2 Caco 2

$148 \mathrm{kDa}$

98 kDa

$64 \mathrm{kDa}$

$50 \mathrm{kDa}$

$36 \mathrm{kDa}$

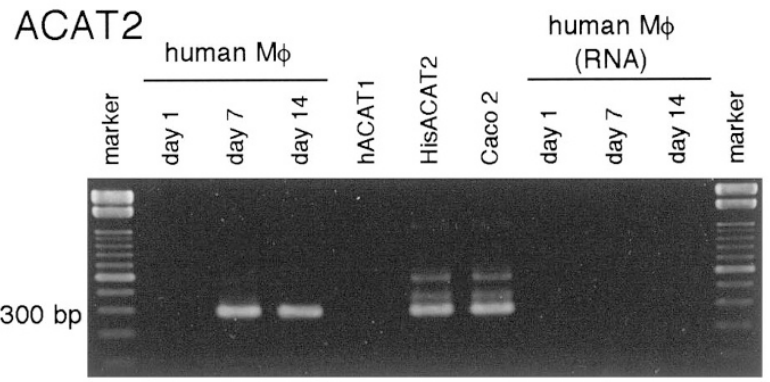

\section{C immunocytochemistry}
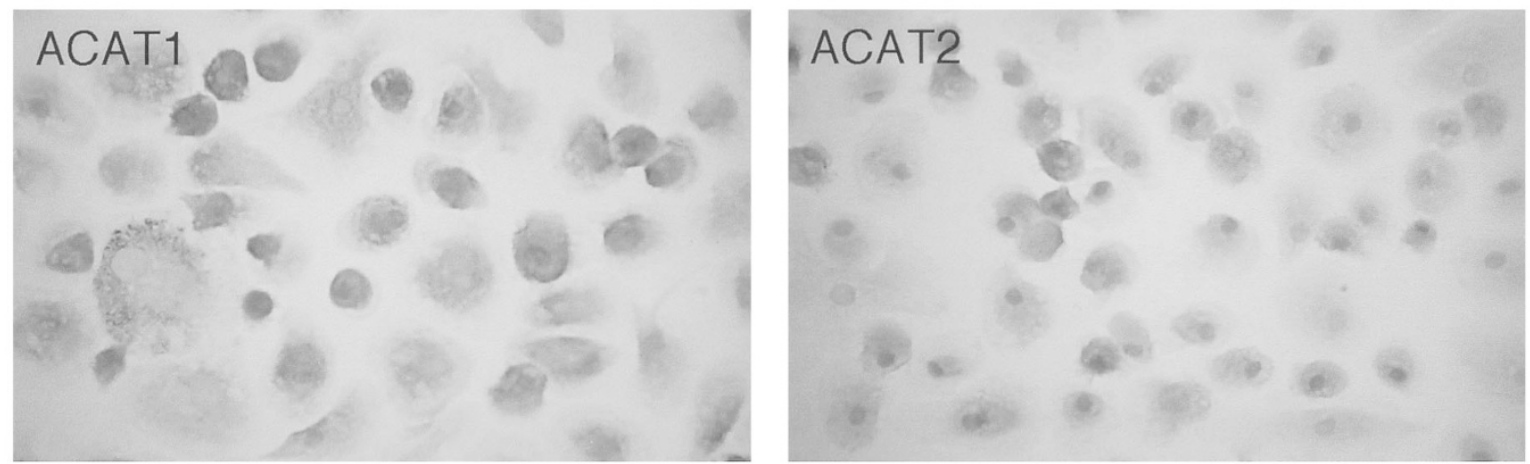

\section{Figure 2.}

In vitro analyses of acyl-coenzyme A:cholesterol acyltransferase 2 (ACAT2) expression in human monocytes-derived macrophages. Immunoblot, RT-PCR, and immunocytochemistry were carried out as described in the "Materials and Methods" section. $\mathrm{M} \phi=$ macrophage. (A) In immunoblot analyses, $100 \mu \mathrm{g}$ of total protein from monocytes-derived macrophages in various levels of differentiation and control cell lines was loaded in each lane. Clear single bands were seen in every experiment for the detection of ACAT1 and ACAT2. These data are representative of nine separate experiments, using monocytes-derived macrophages from individual donors. (B) Each ACAT-isozyme specific RT-PCR signal was shown in different maturation levels of macrophages derived from monocytes and positive control cells lines, hACAT1, HisACAT2, and Caco2 cells. Two $\mu \mathrm{g}$ of total RNA from macrophages and control cells was subjected to reverse transcriptase reaction and proceeded to PCR (ACAT1) or nested PCR reaction (ACAT2). Negative control study was performed by omitting reverse transcriptase reaction, and it gave no signal (human $\mathrm{M} \phi[R N A])$. These data were representative of eight independent experiments. (C) Both ACAT isozymes were also demonstrated by immunocytochemistry in differentiated monocyte-derived macrophages (Day 13). Objective lens used was $\times 40$. 

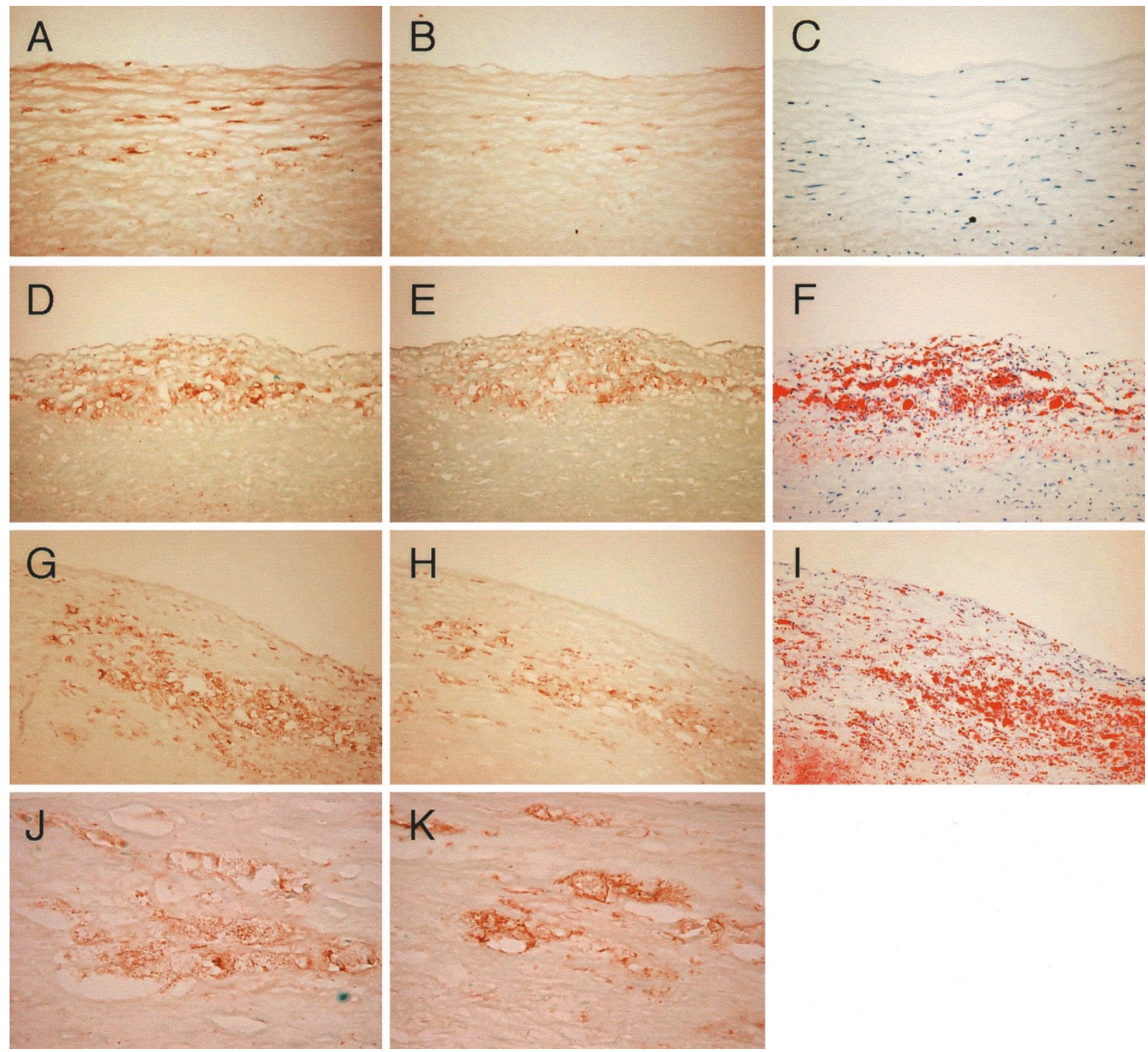

\section{Figure 3.}

Detection of acyl-coenzyme A:cholesterol acyltransferase (ACAT)1- and ACAT2-positive cells in human atherosclerotic aorta. In diffuse intimal thickening lesion (A to $C$ ), a small number of cells were positive for ACAT1 (A) and ACAT2 (B), and no lipid accumulation was observed (C). In more advanced lesions such as diffuse intimal thickening (D to $F)$ and atheromatous plaque ( $G$ to I), the numbers of ACAT1- (D and $G$ ) and ACAT2-positive $(E$ and $H)$ cells were increased, and lipid deposition was more prominent ( $F$ and I). Most of the ACAT isozyme-positive cells were foamy in advanced lesions $(D, E, G, H, J$, and $K$ ), and the distribution of these cells was similar to that of lipid accumulation ( $F$ and I). High power magnification view of ACAT1- $(\mathrm{J}$ ) and ACAT2- $(\mathrm{K})$ positive cells is shown. Objective lenses used were $\times 10$ in $A$ to $C, \times 4$ in $D$ to $I$, and $\times 40$ in $J$ and $K$. These data were representative of 15 independent experiments. Autopsied samples from a separate individual were used for each experiment.

positive cells were foamy, and their distribution was similar to the distribution pattern for lipid deposition in the lesions; however, a small population of ACAT1- or ACAT2-positive cells were lipid free, especially in diffuse intimal thickening lesions (Fig. 3, A to C). Observation by high power magnification revealed that both ACAT isozymes were similarly distributed in the cytoplasm (Fig. 3, J and K).

\section{Double Immunohistochemical Stainings in Human Atherosclerotic Lesions}

It is well known that both macrophages and vascular smooth muscle cells accumulate intracellular cholesteryl esters and subsequently transform to foamy cells (Brown and Goldstein, 1983; Ross, 1986). We had previously demonstrated that in atherosclerotic lesions, the ample expression of ACAT1 only occurs in macrophages but not in smooth muscle cells (Miyazaki et al, 1998). To determine what types of cells express ACAT2 in the atherosclerotic aorta, we performed double immunohistochemical stainings using ACAT2-specific antibodies and antibodies that are specific for marking the cell types. The antibodies that we chose to use, HHF35 (Gown et al, 1986; Tsukada et al, 1987) and KP1 (Facchetti et al, 1988; Pulford et al, 1989), have been routinely used as specific cell markers for vascular smooth muscle cells and for macrophages, respectively. Our results showed that most of the KP1-positive cells were co-stained by antibodies for ACAT2 (Fig. 4, A to D); however, there 

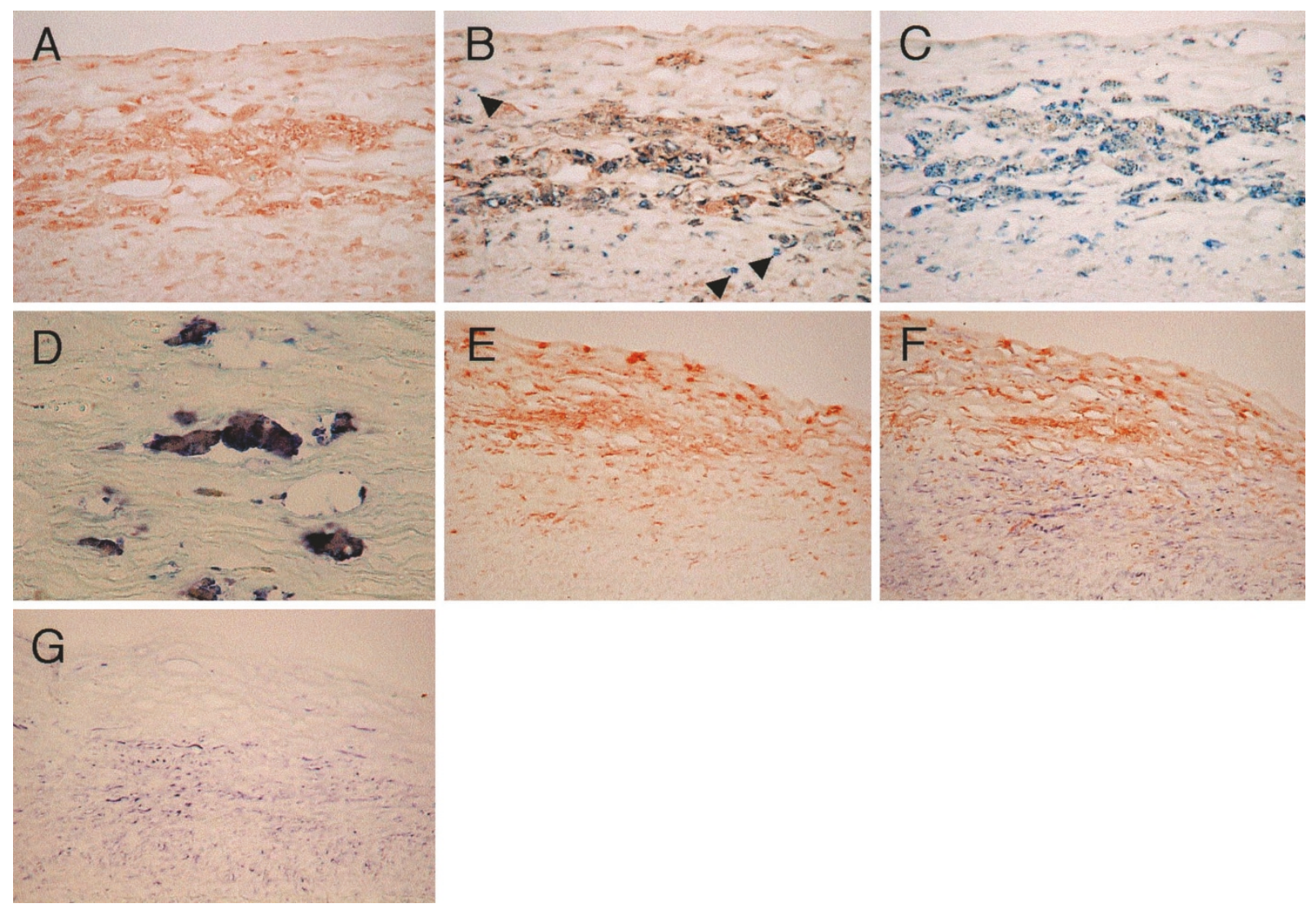

Figure 4.

Determination of the cellular origin of acyl-coenzyme A:cholesterol acyltransferase 2 (ACAT2)-expressing cells in human atherosclerotic aorta. In serial sections of the aorta, the distribution of ACAT2-positive cells (brown color, A) significantly matched that of the CD68-positive cells (blue color, C). Additional double immunohistochemical stainings directly demonstrated that the majority of the CD68-positive cells also expressed ACAT2 (B, lower magnification; D, higher magnification), though a small amount of CD68-positive cells did not express ACAT2 at detectable levels (B, arrowheads). In the human atherosclerotic aorta, the distribution of vascular smooth muscle cells (blue color, G) differed from that of the ACAT2-positive cells (brown color, E); additional double immunohistochemical staining experiment revealed that there were no ACAT2-positive vascular smooth muscle cells in atherosclerotic lesion (F). Objective lenses used were $\times 20$ in A to $\mathrm{C}, \times 40$ in $\mathrm{D}$, and $\times 10$ in $\mathrm{E}$ to $\mathrm{G}$. These data were representative of five independent experiments. Autopsied samples from a separate individual were used for each experiment.

were approximately $20 \%$ to $30 \%$ of the macrophages that stained negative for ACAT2 (Fig. 4B, arrowheads). Previously we have demonstrated that all of the macrophages infiltrated into the lesion express ACATI (Miyazaki et al, 1998). Our current immunoblot data suggest that macrophages with incomplete differentiation express only ACAT1 (Fig. 2A). Therefore, we believe that the reason that not all of the KP1-positive cells in the atherosclerotic human aorta expressed ACAT2 is because KP1 recognizes not only macrophages but also monocytes (Facchetti et al, 1988; Pulford et al, 1989). This interpretation is consistent with our current findings that some but not all of the macrophages in the lesions express ACAT2 and that in the atherosclerotic lesions, fewer cells stained positive for ACAT2 than those for ACAT1 (Fig. 3). Additional experiments showed that no correlation between the ratio of ACAT2-positive cells to the macrophages and the severity of atherosclerosis could be found (data not shown).

We have previously shown that the expression of ACAT1 in smooth muscle cells within the atherosclerotic lesions was essentially undetectable (Miyazaki et al, 1998). To test the possible presence of ACAT2 in smooth muscle cells, we found that nearly all of the ACAT2-expressing cells in the lesions stained negative for the smooth muscle cell marker antibody HHF35 (Fig. 4, E to G).

\section{Detection of ACAT2 mRNA in Human Atherosclerotic Aorta}

To provide additional evidence for the presence of ACAT2-positive macrophages in vivo, we performed RT-PCR analyses using autopsied samples of human atherosclerotic aorta. The results demonstrated the presence of ACAT2 mRNA in these samples (Fig. 5, right panel). As expected, the presence of ACAT1 mRNA was also demonstrated (Fig. 5, left panel)

\section{Detection of ACAT2-Positive Mouse Macrophages Using an In Vitro Assay System}

Using animal models, previous reports indicated that macrophages do not express ACAT2; the grounds for this hypothesis were based on the observation that no 
ACAT1

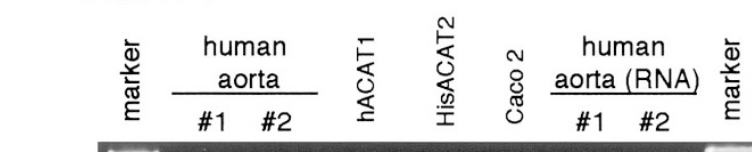

ACAT2

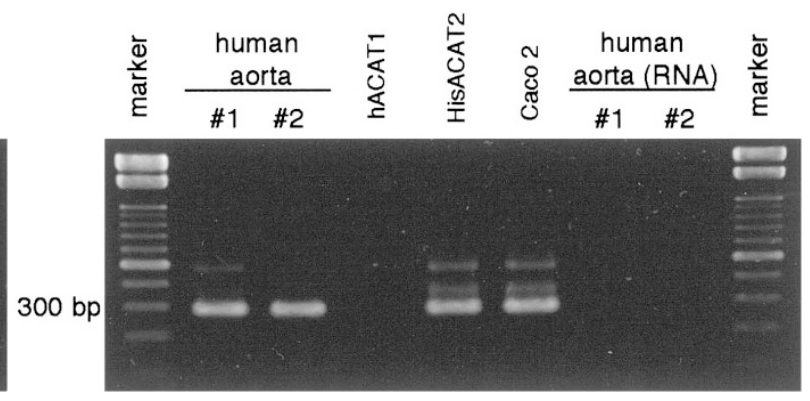

Figure 5 .

Demonstration of acyl-coenzyme A:cholesterol acyltransferase (ACAT) isozyme-specific mRNAs in human atherosclerotic aorta. RT-PCR and nested RT-PCR were carried out as described in the "Materials and Methods" section. Each ACAT-isozyme specific RT-PCR signal was shown in RNA extracts of autopsied human atherosclerotic aorta and positive control cells lines, hACAT1, HisACAT2, and Caco2 cells. One $\mu \mathrm{g}$ of total RNA from tissue samples and control cells was subjected to reverse transcriptase reaction, and proceeded to PCR (ACAT1) or nested PCR (ACAT2) reaction. Negative control study was performed by omitting reverse transcriptase reaction, and it gave no signal (human aorta [RNA]). These data were representative of three independent experiments. Autopsied samples from a separate individual were used for each experiment. The same three samples were used to perform the experiments described in Figure 3.

ACAT2 could be found in peritoneal macrophages or in Kupffer cells (Anderson et al, 1998; Cases et al, 1998; Meiner et al, 1996). After having demonstrated the presence of ACAT2 in human macrophages in pathologic environments, we then tested the possibility that ACAT2 may be present in mouse peritoneal macrophages under chemically induced peritonitis condition. Using both immunoblot and immunocytochemistry methods, we found that although no ACAT2 signal could be detected in resident mouse peritoneal macrophages, clear ACAT2 protein was demonstrated in thioglycollate-elicited mouse peritoneal macrophages (Fig. 6, A and C). To confirm these findings, we showed by RT-PCR analysis that mRNA of ACAT2 was also present in macrophages of mice after thioglycollate treatment (Fig. 6B).

\section{Detection of ACAT2-Positive Macrophages in Mouse Atherosclerotic Aorta}

To test the possibility that ACAT2-positive macrophages may be present in mouse atherosclerotic lesions, immunohistochemical stainings with ACAT2specific antibodies were performed using Apo E-/mice. The results showed that both ACAT1- and ACAT2-positive cells were found in mouse atheromatous lesions (Fig. 7A); these cells were stained positive by the macrophage specific-marker antibody BM8 (data not shown). Lipid accumulation was prominent in cells that express the ACAT1 and ACAT2 signals (Fig. $7 A$, oil red $O)$. Besides immunostaining we also demonstrated that there was detectable mouse ACAT2 mRNA in Apo E-/- aorta (Fig. 7B). These data show that both ACAT1 and ACAT2 are present in macrophages in the atherosclerotic aorta of hyperlipidemic mice.

\section{Discussion}

Until now it was believed that the tissue distribution of ACAT1 was ubiquitous, while that of ACAT2 was restricted to intestinal enterocytes and liver hepatocytes only (Anderson et al, 1998; Buhman et al, 2000;
Cases et al, 1998; Chang et al, 2000, 2001; Joyce et al, 1999; Lee et al, 2000; Oelkers et al, 1998; Pape et al, 1995). In this report, using a specific anti-ACAT2 antibody and highly sensitive immunostaining and RT-PCR methods, we show that ACAT2 is clearly expressed in macrophages, indicating that the ACAT2 distribution is not intestine and liver specific. Therefore, ACAT2 plays a functional role in macrophages in addition to its role in the intestines and liver. Our current work will have an important impact on formulating the appropriate concept for developing isozyme-specific ACAT inhibitors to treat hyperlipidemia and atherosclerosis.

We also show that in humans and in mice, ACAT2 expression becomes detectable only in macrophages under various pathologic environments but not in resident macrophages in physiologic conditions. This finding may explain why other investigators have failed to detect the existence of ACAT2 in macrophages under normal circumstances. Our results can also explain the finding by Yagyu et al (2000), who showed that decreased but detectable cholesterol esters were present in the atherosclerotic lesions of ACAT1-/ -:Apo E-/- or ACAT1-/-:LDLR-/- double knockout mice. It also explains the results of Lee et al (1998), who showed that anti-ACAT1 antibodies could not immunodeplete more than $80 \%$ of the total ACAT activity present in extracts of human macrophages. At present, we do not know the relative content, subcellular distribution, or the exact function or functions of ACAT1 and ACAT2 in human macrophages, nor can we explain why both isoenzymes should be present in a single cell type. It is tempting to hypothesize that the roles of ACAT1 and ACAT2 in macrophages may be complementary to each other to provide optimal functions of the fully differentiated macrophages. Because both the resident tissue macrophages and macrophages under pathologic conditions express ACAT1, it is possible that in macrophages the expression of ACAT1 may be nearly constitutive, while the expression of ACAT2 may be more inducible; ACAT2 expression may be more critically dependent on specific 


\section{A immunoblot}

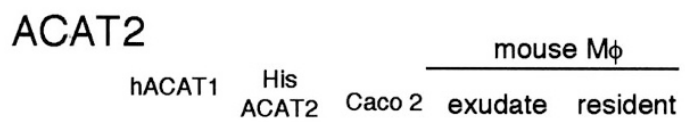

148 kDa

$98 \mathrm{kDa}$

64 kDa

$50 \mathrm{kDa}$

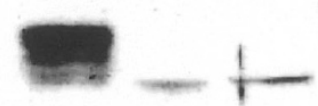

$36 \mathrm{kDa}$

\section{B. RT-PCR}

ACAT2
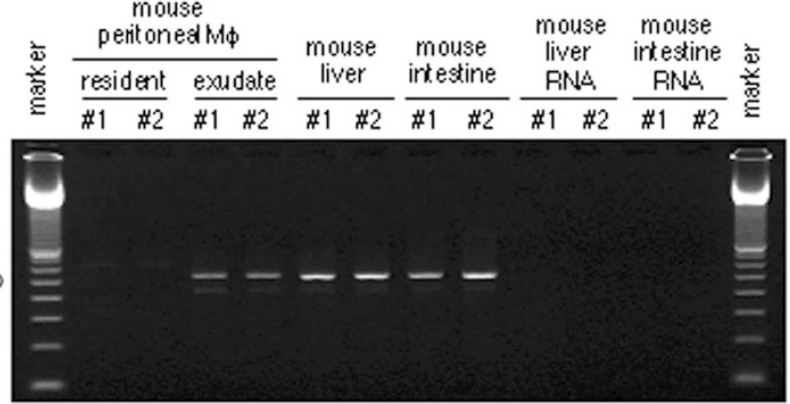

\section{C immunocytochemistry}
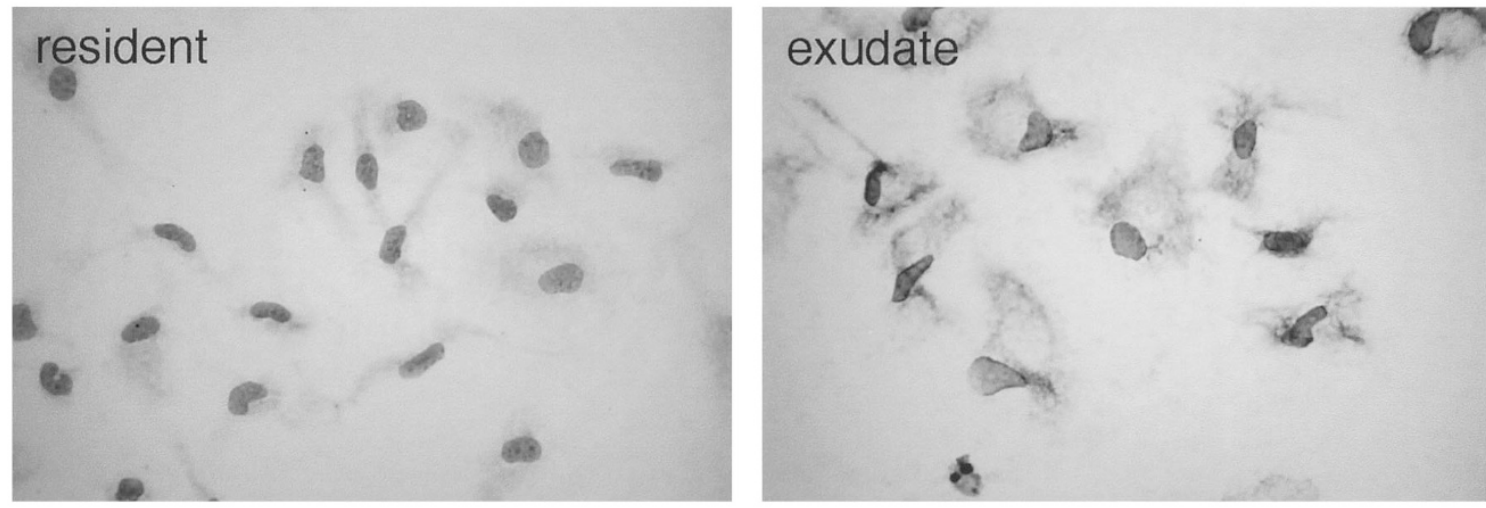

Figure 6.

In vitro analysis of acyl-coenzyme A:cholesterol acyltransferase 2 (ACAT2) expression in mouse peritoneal macrophages. Immunoblot, RT-PCR, and immunocytochemistry were performed as described in the "Materials and Methods" section. $M \phi=$ macrophage. (A) In immunoblot analysis, $100 \mu \mathrm{g}$ of crude cell extract proteins from each sample was loaded onto each lane. A clear 46-kd ACAT2 signal was shown in thioglycollate-treated mouse peritoneal macrophages (exudate), whereas no signal was demonstrated in untreated macrophages (resident). In control cell lines, no signals were seen in hACAT1. Two signals, major 50-kd signals from histidine tagged ACAT2 and minor 46-kd ACAT2 signal, were observed in HisACAT2, and 46-kd signal was shown in Caco2 cells. This result was representative of six separate experiments. (B) For the detection of ACAT2 mRNA in mouse macrophages, $1 \mu \mathrm{g}$ of extracted total RNA was proceeded to reverse transcriptase reaction and subsequently subjected to PCR reaction. Clear ACAT2-specific 530-bp RT-PCR signals were shown in thioglycollate-treated macrophages (exudate) and positive control samples, mouse liver, and small intestine RNA extracts. These data were representative of five independent RT-PCR experiments. Signal from sample omitting reverse transcriptase reaction was not detected (mouse liver RNA and mouse intestine RNA). (C) ACAT2 in mouse peritoneal macrophages with thioglycollatetreatment were also detected immunocytochemically but not in those without treatments. Hematoxylin was employed as counter stain dye. Objective lens used was $\times 40$.

signals elicited under various pathophysiologic conditions. The signals that up-regulate the expressions of ACAT1 in monocytes-derived macrophage include steroid hormones such as dexamethasone, interferon- $\gamma$, and vitamin $D_{3}$ (Cheng et al, 1995;
Maung et al, 2001; Panousis and Zuckerman, 2000; Yang et al, 2001). In the future, it will be interesting to find out whether these or other agents can up-regulate the ACAT2 expression in macrophages. In mice it would be particularly interesting to find out 


\section{A. immunohistochemistry}
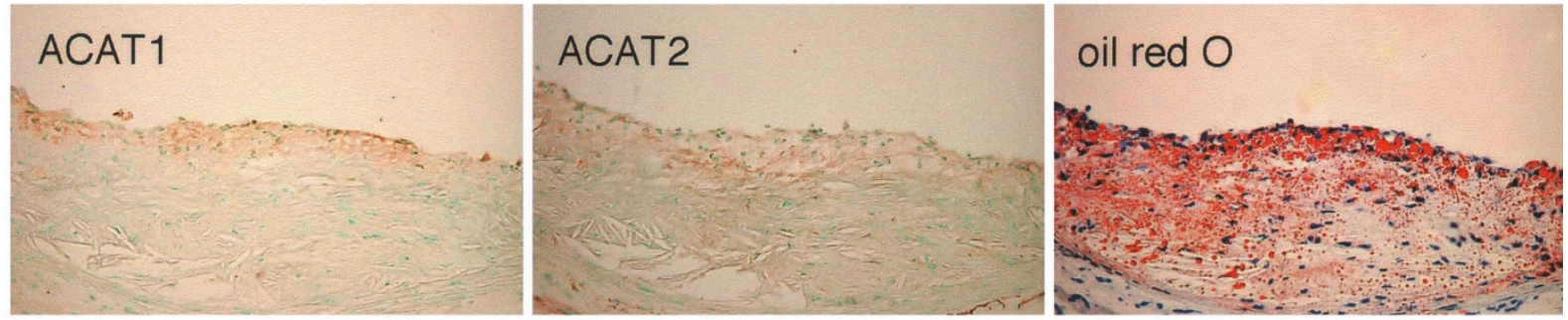

\section{B. RT-PCR}

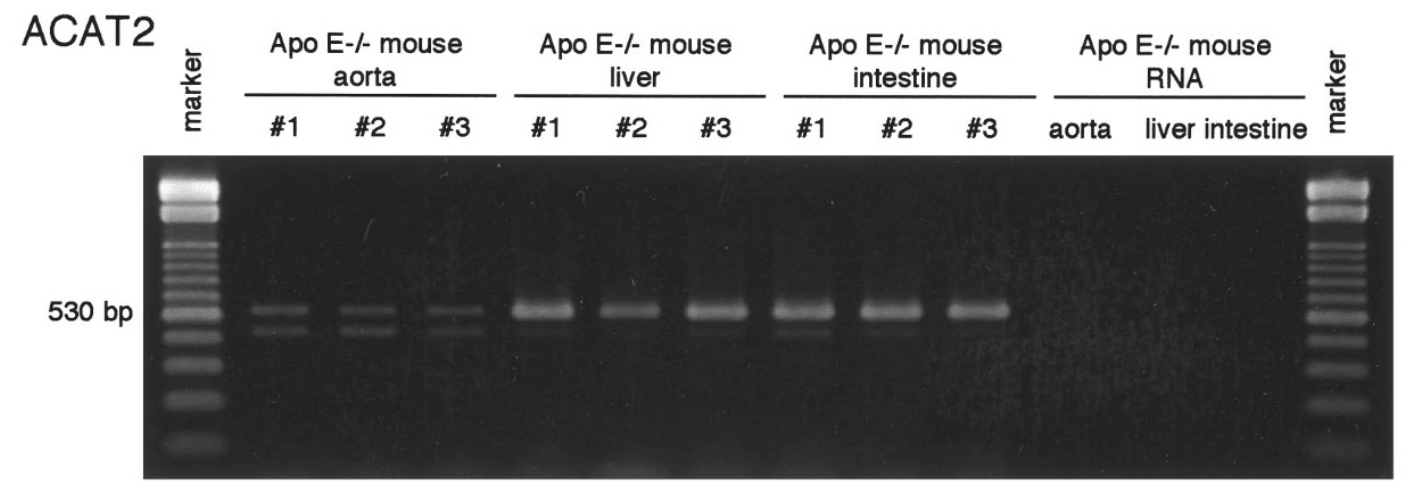

Figure 7.

Demonstration of acyl-coenzyme A:cholesterol acyltransferase 2 (ACAT2) expression in mouse atherosclerotic aorta. (A) Immunostain demonstrating two ACAT isozymes (ACAT1 and ACAT2) expression and lipid accumulation in the lesion. All stainings were performed using Apo $\mathrm{E}-/-$ mice fed a Western-style diet. Objective lens used was $\times 10$. (B) To detect ACAT2 mRNA in mouse atherosclerotic tissues, $1 \mu \mathrm{g}$ of extracted total RNA from Apo E-/ - tissue samples underwent reverse transcriptase reaction and was subsequently subjected to PCR reaction. Mouse ACAT2-specific 530-bp RT-PCR signals were shown in the aortas with atherosclerosis and positive control samples (liver and intestine). Signal from sample omitting reverse transcriptase reaction was not detected (mouse liver RNA and mouse intestine RNA).

whether the ACAT2 gene in macrophages is induced under conditions where the ACAT1 gene has been deleted. The expression of ACAT2 may not be restricted to intestines, liver, and macrophages only, and it will also be interesting to examine the possible ACAT2 expression in various other tissues under different pathophysiologic conditions.

\section{Materials and Methods}

\section{Tissue Preparation}

Unless stated otherwise, human tissue samples for the current study were collected from autopsy within a 3-hour postmortem period. The specimens from autopsy were fixed by $2 \%$ of periodate-lysineparaformaldehyde for 4 to 6 hours, and $15 \%$ of formalin was used as a fixative for other histologic samples. In the frozen section, fixed tissue samples were washed by PBS containing a graded series of saccharose $(10 \%, 15 \%$, and $20 \%)$ for 5 to 12 hours each, embedded in Tissue-Tek O.C.T. compound (Sakura Finetechnical Company, Tokyo, Japan), and frozen by liquid nitrogen. They were cut into $5-\mu \mathrm{m}-$ thick sections by a cryostat (HM500M, MICROM International $\mathrm{GmbH}$, Walldorf, Germany) and stored at $-80^{\circ} \mathrm{C}$ until use. To intensify the immunoreactivity, all paraffin-embedded samples were immersed in $95^{\circ} \mathrm{C}$ citrate buffer ( $\mathrm{pH}$ 6.0) for 10 minutes after deparaffinization, and then immunostaining was performed.

The mouse aorta with atheromatous plaque was obtained from Apo $\mathrm{E}-\mathrm{/}-$ mice fed an 8-week Western-style diet. The aorta was removed, fixed with $2 \%$ periodate-lysine-paraformaldehyde, and prepared as frozen sections for histologic examinations as described above.

For RT-PCR experiments, the human aorta with atheromatous plaque and Apo $\mathrm{E}-/-$ mice tissue samples (liver, intestine, and atherosclerotic aorta) were removed and rapidly frozen using liquid nitrogen, and stored at $-80^{\circ} \mathrm{C}$ until use.

All of the experiments described in this study have been pre-approved by the ethical committee at Kumamoto University School of Medicine, Kumamoto, Japan.

\section{Antibodies}

The specific rabbit polyclonal antibody against the human ACAT1 (DM10) was described previously (Chang et al, 1995). We used the high-titer rabbit polyclonal anti-ACAT2 antibody DM54 (Chang et al, 2000) to perform all of the experiments involving ACAT2 reported in the current work. The specificity of DM54 in recognizing human ACAT2 in human livers 
and human intestines has been demonstrated in our previous work (Chang et al, 2000) by using several techniques that include immunoprecipitation, histochemical staining, and Western blotting; the criteria for specificity have been documented. In addition, the specificity of DM54 in recognizing human ACAT2 in human macrophages is demonstrated in Figure $2 \mathrm{~A}$. As shown in our previous work (Chang et al, 2000), and in Figure 2, human ACAT2 is a 46-kd protein on sodium dodecyl sulfate-polyacrylamide gel electrophoresis (SDS-PAGE). In results not shown, we used liver microsomes prepared from normal mice and ACAT2 gene knockout (ACAT2-/-) mice, and then used DM54 antibodies to perform Western blotting analysis. The liver microsomes of the ACAT2-/- mice (Buhman et al, 2000) were kindly provided by the laboratory of Dr. Robert Farese of the University of California, San Francisco. The results showed that DM54 recognized a single 46-kd band in liver samples from the normal mice; this band was clearly absent in liver samples from the ACAT2-/- mice. Therefore, DM54 is a specific antibody that recognizes mouse ACAT2.

Mouse monoclonal antibodies for human monocytes/macrophage marker CD68 (KP1) and for vascular smooth muscle marker actin (HHF35) were purchased from Dako Japan (Kyoto, Japan).

\section{Immunostaining and Histochemistry}

The indirect dextran-polymer conjugated immunoperoxidase method was employed for detection of ACAT2 in tissue samples. Briefly, after inhibition of endogenous peroxidase activity by method of Isobe et al (1977), the specimens were incubated with 5\% normal goat serum for 20 minutes and subsequently reacted with DM54, of which the final Ig concentration was $8 \mu \mathrm{g} / \mathrm{ml}$, for 60 minutes as a primary antibody. After washing out excess antibodies with PBS $(\mathrm{pH} 7.2)$ at $4^{\circ} \mathrm{C}$, the samples were incubated with dextranpolymer conjugated horseradish peroxidase-labeled goat antirabbit antibody (Envision Plus, Dako Japan, Kyoto, Japan) as a secondary antibody for 60 minutes. Immunoreaction was visualized by DAB + Liquid System (Dako Japan, Kyoto, Japan) after rinsing excess secondary antibodies, which gave a brown color to the immunoreactants. To inhibit a nonspecific reaction, $0.1 \%$ of Tween 20 (Nakalai Tesque, Kyoto, Japan) was added into buffers for dilution of the primary antibody and sample washing. Specific immunoreactivity was confirmed by setting adequate negative control using nonimmunized rabbit Ig as a primary antibody. In immunostainings for ACAT1, the conventional indirect immunoperoxidase method was employed as described previously (Sakashita et al, 2000).

For immunocytochemistry, the cultured human and mice macrophages were fixed with $2 \%$ of periodatelysine-paraformaldehyde for 20 minutes at $4^{\circ} \mathrm{C}$ and washed three times with ice-cold phosphate-buffered saline containing $0.05 \%$ of saponin (Sigma, St. Louis, Missouri) for 5 minutes each time. After rinsing out excessive saponin, immunostaining was carried out as described above. Oil red O staining was employed to detect lipid accumulation in the serial sections of tissue samples using immunohistochemistry.

\section{Cell Culture}

Human cultured macrophages derived from bloodborne monocytes were collected from healthy volunteers using the method described elsewhere (Sakai et al, 1996). Briefly, mononuclear leukocytes were segregated from peripheral blood by the Ficoll/Hypaque gradient centrifugation method using Mono-Poly Resolving Medium (Dainippon Pharmaceutical Company, Osaka, Japan). Collected blood cells were resuspended in RPMI medium 1640 (GIBCO BRL Life Technologies, Rockville, Maryland) supplemented by $10 \%$ human serum (CELLECT human type $A B$ serum, ICN Biomedicals, Aurora, Ohio), $0.1 \mathrm{mg} / \mathrm{ml}$ of streptomycin, and 100 unit/ml of penicillin. The $1 \times 10^{7}$ mononuclear cells were plated in $10-\mathrm{cm}$ plastic dishes for 2 hours at $37^{\circ} \mathrm{C}$, and nonadherent cells were removed by gentle washing with culture medium. The remained monocytes (about $1 \times 10^{6}$ cells per dish) were cultured up to 14 days to induce differentiation and maturation into macrophages. The monocytes and macrophages under various differentiation levels were withdrawn at Days 1, 4, 7, 10, and 14, and stored at $-80^{\circ} \mathrm{C}$ until use.

For preparation of mouse peritoneal macrophages, male C57BL/6 mice were injected with $4 \mathrm{ml}$ of thioglycollate (Difco Laboratories, Detroit, Michigan) into the peritoneal cavity, and peritoneal macrophages were harvested 4 days after injection. The cells were washed twice by RPMI medium 1640 containing 10\% FCS (HyClone Laboratories Inc., Logan, Utah), and incubated for 1 hour to let them adhere to the $10-\mathrm{cm}$ culture dishes. After removal of nonadherent cells by a gentle wash, the cells were subjected to immunoblot or RT-PCR experiments.

To serve as a positive control for immunoblot and RT-PCR experiments, Caco2 (Chang et al, 1995, 2000), hACAT1, and HisACAT2 (Chang et al, 2000) cells were prepared. The $\mathrm{Caco} 2$ cells were cultured with DMEM (GIBCO BRL Life Technologies) containing antibiotics and $10 \%$ of FCS, and harvested 10 to 14 days after their confluent growth. Both hACAT1 and HisACAT2 cells were grown in F12 Nutrient Mixture (GIBCO BRL Life Technologies) containing 10\% FCS and antibiotics, and harvested after preconfluent growth. All of these cells were washed with ice-cold phosphate-buffered saline twice and subjected to each experiment.

\section{Immunoblot}

Determination of protein concentration, SDS-PAGE, and immunoblot analyses were performed as described elsewhere (Chang et al, 1995, 1998, 2000). Stored macrophages and control cell lines were thawed and extracted with $0.1 \mathrm{ml}$ of $10 \%$ SDS containing the protease inhibitors $(0.2 \mathrm{mmol} / \mathrm{l}$ of $4-(2-$ aminoethyl)-benzenesulfonyl fluoride, $1 \mu \mathrm{g} / \mathrm{ml}$ of leu- 
Table 1. The Primer Pairs used in RT-PCR Experiments

\begin{tabular}{|c|c|c|c|}
\hline Primers & Sense/antisense & Sequences & Size of PCR products \\
\hline \multirow[t]{2}{*}{ Human ACAT1 } & Sense & 5'-CGGAATATCAAACAGGAGCCCTTC-3' & 405 bp \\
\hline & Antisense & $5^{\prime}-$ CATTCCAAAGAACATGAAGAGCACG-3' & \\
\hline \multirow[t]{2}{*}{ Human ACAT2 (outer primer) } & Sense & $5^{\prime}-$ TTTCTCCAGCTACCTCTAC-3' & 546 bp \\
\hline & Antisense & $5^{\prime}-$ ATGACGGGATAGAAGAACC $-3^{\prime}$ & \\
\hline \multirow[t]{2}{*}{ Human ACAT2 (inner primer) } & Sense & 5'-CCTAGGACGCCCTATGTCAG-3' & 300 bp \\
\hline & Antisense & 5'-GGACGTTGAGTTCCACCAGT-3' & \\
\hline \multirow[t]{2}{*}{ Mouse ACAT2 } & Sense & 5'-TTTGCTCTATGCCTGCTTCA-3' & 530 bp \\
\hline & Antisense & $5^{\prime}-$ CCATGAAGAGAAAGGTCCACA-3' & \\
\hline
\end{tabular}

ACAT, acyl-coenzyme A:cholesterol acyltransferase.

peptin, $1 \mu \mathrm{g} / \mathrm{ml}$ of pepstatin A, and $4 \mu \mathrm{g} / \mathrm{ml}$ of aprotinin, and $2 \mathrm{mmol} / \mathrm{l}$ of etylenediaminetetraacetic acid (all were purchased from Sigma Chemical Company). To prevent protein aggregation, $50 \mathrm{mmol} / \mathrm{l}$ of dithiothreitol was added to the cell lysis buffer. Cells were scraped and sheared, using syringes with 25gauge needles. Samples were run on $10 \%$ SDS-PAGE and subjected to immunoblotting. The primary antibodies (DM10 and DM54) were used at a final Ig concentration of $0.2 \mu \mathrm{g} / \mathrm{ml}$. Quantification of both ACAT signals was performed using LAS1000plus system (Fuji Film, Tokyo, Japan).

\section{Double Immunohistochemical Staining}

The samples were immunostained by ACAT2-specific rabbit polyclonal antibodies as described in the "Immunohistochemistry and Histochemistry" section, and excess substrate was rinsed away. The specimens were subsequently incubated to each cell-type specific mouse mAb (KP1 or HHF35) as second step immunostaining. To visualize following immunoreaction, we used alkaline phosphatase anti-alkaline phosphatase method as described previously (Miyazaki et al, 1998); using naphthol AS-MX phospate and fast blue BB salt as substrates for alkaline phosphatase, this step gave a blue color to the immunoreaction. The negative control for the immunoreaction was performed by using rabbit and mouse nonimmune Ig as primary antibodies in each immunostaining step.

\section{RT-PCR}

Total RNA was extracted from human and mouse macrophages, from Caco2, hACAT1, HisACAT2 cells, from mouse liver, intestine, and from atherosclerotic human and Apo $\mathrm{E}-/-$ mouse aortas using RNeasy Mini Kit (Qiagen $\mathrm{GmBH}$, Hilden, Germany). The samples were subjected to reverse transcriptase reaction using a random primer, and PCR reaction was performed as described elsewhere (Hagiwara et al, 1999). The primer pairs for human ACAT1 and ACAT2 and mouse ACAT2 used in these experiments are summarized in Table 1. The PCR mixtures of human ACAT1 and mouse ACAT2 were conditioned using HotStarTaq PCR System (Qiagen GmBH, Hilden, Germany), and amplified by a Gene Amplification PCR System 2400 (Perkin-Elmer, Norwalk, Connecticut). PCR con- ditioned samples were first incubated at $95^{\circ} \mathrm{C}$ for 15 minutes and then cycled 35 times at $94^{\circ} \mathrm{C}$ for 30 seconds, at $56^{\circ} \mathrm{C}$ for 30 seconds, at $72^{\circ} \mathrm{C}$ for 1 minute, and finally incubated at $72^{\circ} \mathrm{C}$ for 10 minutes. For the detection of ACAT2 signals in human cultured macrophages and atherosclerotic aorta, we employed nested PCR system. The PCR mixtures containing TaqDNA polymerase (Invitrogen Corporotion, Carlsbad, California) were subjected to the PCR reaction using human ACAT2 outer primers (PCR condition was 3 minutes at $94^{\circ} \mathrm{C}, 30$ cycles of 50 seconds at $94^{\circ} \mathrm{C}, 30$ seconds at $55^{\circ} \mathrm{C}, 1$ minute at $72^{\circ} \mathrm{C}$, followed by a final extension step of 10 minutes at $72^{\circ} \mathrm{C}$ ), and sequentially subjected to nested PCR reaction using human ACAT2 inner primers (PCR condition was 3 minutes at $94^{\circ} \mathrm{C}, 30$ cycles of 50 seconds at $94^{\circ} \mathrm{C}, 30$ seconds at $58^{\circ} \mathrm{C}, 1$ minute at $72^{\circ} \mathrm{C}$, followed by a final extension step of 10 minutes at $72^{\circ} \mathrm{C}$ ). The PCR products were run on $2 \%$ agarose gel and were visualized by ultraviolet transillumination after nucleic acid gel stain using SYBER Green (BioWhittaker Molecular Applications, Rockland, Maine).

\section{References}

Accad M, Smith SJ, Newland DL, Sanan DA, King LE, Linton MF, Fazio S, and Farese RV (2000). Massive xanthomatosis and altered composition of atherosclerotic lesions in hyperlipidemic mice lacking acyl CoA:cholesterol acyltransferase 1. J Clin Invest 105:711-719.

Anderson RA, Joyce C, Davis M, Reagan JW, Clark M, Shelness GS, and Rudel LL (1998). Identification of a form of acyl-CoA:cholesterol acyltransferase specific to liver and intestine in nonhuman primate. J Biol Chem 273:2674726754.

Brown MS and Goldstein JL (1983). Lipoprotein metabolism in the macrophage: Implications for cholesterol deposition in atherosclerosis. Annu Rev Biochem 52:223-261.

Buhman KK, Accad M, Novak S, Choi RS, Wong JS, Hamilton RL, Turley S, and Farese RV Jr (2000). Resistance to diet-induced hypercholesterolemia and gallstone formation in ACAT2-deficient mice. Nat Med 6:1341-1347.

Cases S, Novak S, Zheng YW, Myers HM, Lear SR, Sande E, Welch CB, Lusis AJ, Spencer TA, Krause BR, Erickson SK, and Farese RV Jr (1998). ACAT-2, a second mammalian acyl-CoA:cholesterol acyltransferase: Its cloning, expression and characterization. J Biol Chem 273:26755-26764. 
Chang CC, Chen J, Thomas MA, Cheng D, Del Priore VA, Newton RS, Pape ME, and Chang TY (1995). Regulation and immunolocalization of acyl-coenzyme A: cholesterol acyltransferase in mammalian cells as studied with specific antibodies. J Biol Chem 270:29532-29540.

Chang CC, Lee CY, Chang ET, Cruz JC, Levesque MC, and Chang TY (1998). Recombinant acyl-CoA:cholesterol acyltransferase-1 (ACAT-1) purified to essential homogeneity utilizes cholesterol in mixed micelles or in vesicles in a highly cooperative manner. J Biol Chem 273:35132-35141.

Chang CCY, Huh HY, Cadigan KM, and Chang TY (1993). Molecular cloning and functional expression of human acylcoenzyme A:cholesterol acyltransferase cDNA in mutant Chinese hamster ovary cells. J Biol Chem 268:20747-20755.

Chang CCY, Sakashita N, Ornvold K, Lee O, Chang ET, Dong R, Lin S, Lee Chi-Yu G, Strom SC, Kashyap R, Fung JJ, Farase RV Jr., Patoiseau J-F, Delhon A, and Chang TY (2000). Immunological quantitation and localization of ACAT-1 and ACAT-1 in human liver and small intestine. J Biol Chem 275:28083-28092.

Chang TY, Chang CC, Lin S, Yu C, Li BL, and Miyazaki A (2001). Roles of acyl-coenzyme A:cholesterol acyltransferase-1 and -2. Curr Opin Lipidol 12:289-296.

Chang TY, Chang CCY, and Cheng D (1997). Acyl-coenzyme A:cholesterol acyltransferase. Annu Rev Biochem 66:613638.

Cheng W, Kvilekval KV, and Abumrad NA (1995). Dexamethasone enhances accumulation of cholesteryl esters by human macrophages. Am J Physiol 269:E642-E648.

Facchetti F, de Wolf-Peeters C, Mason DY, Pulford K, van den Oord JJ, and Desmet VJ (1988). Plasmacytoid T cells. Immunohistochemical evidence for their monocyte/macrophage origin. Am J Pathol 133:15-21.

Gown AM, Tsukada T, and Ross R (1986). Human atherosclerosis. II. Immunocytochemical analysis of the cellular composition of human atherosclerotic lesions. Am J Pathol 125:191-207.

Hagiwara SI, Takeya M, Suzuki H, Kodama T, van der Laan LJ, Kraal G, Kitamura N, and Takahashi K (1999). Role of macrophage scavenger receptors in hepatic granuloma formation in mice. Am J Pathol 154:705-720.

Isobe S, Nakane PK, and Brown WR (1977). Studies on translocation of immunoglobulins across intestinal epithelium. I. Improvements in the peroxidase-labeled antibody method for application to study of human intestinal mucosa Acta Histochem Cytochem 10:161-171.

Johnson WD, Mei B, and Cohn ZA (1977). The separation, long-term cultivation, and maturation of the human monocyte. J Exp Med 146:1613-1626.

Joyce C, Skinner K, Anderson RA, and Rudel LL (1999). Acyl-coenzyme A:cholesteryl acyltransferase 2. Curr Opin Lipidol 10:89-95.

Lee O, Chang CCY, Lee W, and Chang TY (1998). Immmunodepletion experiments suggest that acyl-coenzyme A:cholesterol acyltransferase-1 (ACAT-1) protein plays a major catalytic role in adult human liver, adrenal gland, macrophages, and kidney, but not intestine. J Lipid Res 39:17221727.

Lee RG, Willingham MC, Davis MA, Skinner KA, and Rudel LL (2000). Differential expression of ACAT1 and ACAT2 among cells within liver, intestine, kidney and adrenal of nonhuman primates. J Lipid Res 41:1991-2001.

Matsuda K (1994). ACAT inhibitors as antiatherosclerotic agents: Compounds and mechanisms. Med Res Rev 14:271305.

Maung KK, Miyazaki A, Nomiyama $\mathrm{H}$, Chang CCY, Chang TY, and Horiuchi S (2001). Induction of acyl-coenzyme A:cholesterol acyltrasferase- 1 by 1,25 -dihydroxyvitamin $D_{3}$ or 9-cis-retinoic acid in undifferentiated THP-1 cells. J Lipid Res 42:181-187.

Meiner VL, Cases S, Myers HM, Sande ER, Bellosta S, Schambelan M, Pitas RE, McGuire J, Herz J, and Farese RV Jr (1996). Disruption of the acyl-CoA:cholesterol acyltransferase gene in mice: Evidence suggesting multiple cholesterol esterification enzymes in mammals. Proc Natl Acad Sci USA 93:14041-14046.

Miyazaki A, Sakashita N, Lee O, Takahashi K, Horiuchi S, Hakamata H, Morganelli PM, Chang CC, and Chang TY (1998). Expression of ACAT-1 protein in human atherosclerotic lesions and cultured human monocytes-macrophages. Arterioscler Thromb Vasc Biol 18:1568-1574.

Oelkers P, Behari A, Cromley D, Billheimer JT, and Sturley SL (1998). Characterization of two human genes encoding acyl coenzyme A:cholesterol acyltransferase-related enzymes. J Biol Chem 273:26765-26771.

Panousis CG and Zuckerman SH (2000). Interferon-gamma induces downregulation of Tangier disease gene (ATPbinding cassette transporter 1) in macrophage-derived foam cells. Arterioscler Thromb Vasc Biol 20:1423-1424.

Pape ME, Schultz PA, Rea TJ, DeMattos RB, Kieft K, Bisgaier CL, Newton RS, and Krause BR (1995). Tissue specific changes in acyl-CoA:cholesterol acyltransferase (ACAT) mRNA levels in rabbits. J Lipid Res 36:823-838.

Pulford KA, Rigney EM, Micklem KJ, Jones M, Stross WP, Gatter KC, and Mason DY (1989). KP1: A new monoclonal antibody that detects a monocyte/macrophage associated antigen in routinely processed tissue sections. J Clin Pathol 42:414-421.

Ross R (1986). The pathogenesis of atherosclerosis: An update. N Engl J Med 314:448-500.

Sakai M, Miyazaki A, Hakamata H, Sato Y, Matsumura T, Kobori S, Shichiri M, and Horiuchi S (1996). Lysophosphatidylcholine potentiates the mitogenic activity of modified LDL for human monocyte-derived macrophages. Arterioscler Thromb Vasc Biol 16:600-605.

Sakashita N, Miyazaki A, Takeya M, Horiuchi S, Chang CC, Chang TY, and Takahashi K (2000). Localization of human acyl-coenzyme A: cholesterol acyltransferase-1 (ACAT-1) in macrophages and in various tissues. Am J Pathol 156:227236.

Tabas I (1995). The stimulation of the cholesterol esterification pathway by atherogenic lipoproteins in macrophages. Curr Opin Lipidol 6:260-268.

Tsukada T, Tippens D, Gordon D, Ross R, and Gown AM (1987). HHF35, a muscle-actin-specific monoclonal antibody. I. Immunocytochemical and biochemical characterization Am J Pathol 126:51-60.

Yagyu H, Kitamine T, Osuga J, Tozawa R, Chen Z, Kaji Y, Oka T, Perry S, Tamura Y, Ohashi K, Okazaki H, Yahagi H, Shionoiri F, lizuka Y, Harada K, Shimano H, Yamashita H, Gotoda T, Yamada N, and Ishibashi S (2000). Absence of 
ACAT-1 attenuates atherosclerosis but causes dry eye and cutaneous xanthomatosis in mice with congenital hyperlipidemia. J Biol Chem 275:21324-21330.

Yang JB, Duan ZJ, Yao W, Lee O, Yang L, Yang XY, Sun X, Chang CC, Chang TY, and Li BL (2001). Synergistic transcriptional activation of human Acyl-coenzyme A:cholesterol acyltransferase-1 gene by interferon-gamma and all-transretinoic acid THP-1 cells. J Biol Chem 276:20989-20998.
Zuckerman SH, Ackerman SK, and Douglas SD (1979). Long-term human peripheral blood monocyte cultures: Establishment, metabolism and morphology of primary human monocyte-macrophage cell cultures. Immunology 38:401411. 